\title{
Sex-Specific Effects of High Yolk Androgen Levels on Constitutive and Cell-Mediated Immune Responses in Nestlings of an Altricial Passerine ${ }^{\star}$
}

\author{
Jaime Muriel ${ }^{1, \dagger}$ \\ Lorenzo Pérez-Rodríguez ${ }^{2}$ \\ Manuel E. Ortiz-Santaliestra ${ }^{3}$ \\ Marisa Puerta ${ }^{4}$ \\ Diego Gil ${ }^{1}$ \\ ${ }^{1}$ Departamento de Ecología Evolutiva, Museo Nacional de \\ Ciencias Naturales (MNCN-Consejo Superior de \\ Investigaciones Científicas [CSIC]), José Gutiérrez Abascal 2, \\ E-28006 Madrid, Spain; ${ }^{2}$ Departamento de Ecología Evolutiva, \\ Estación Biológica de Doñana (EBD-CSIC), Avenida Américo \\ Vespucio s/n, Isla de la Cartuja, 41092 Seville, Spain; ${ }^{3}$ Instituto \\ de Investigación en Recursos Cinegéticos (IREC-CSIC- \\ Universidad de Castilla-La Mancha [UCLM]-Junta de \\ Comunidades de Castilla-La Mancha [JCCM]), Ronda de \\ Toledo s/n, 13071 Ciudad Real, Spain; ${ }^{4}$ Departamento de \\ Fisiología Animal II, Facultad de Ciencias Biológicas, \\ Universidad Complutense, José Antonio Novais 2, Ciudad \\ Universitaria, 28040 Madrid, Spain
}

Accepted 7/8/2016; Electronically Published 8/29/2016

\section{ABSTRACT}

Avian embryos are exposed to yolk androgens that are incorporated into the egg by the ovulating female. These steroids can affect several aspects of embryo development, often resulting in increases in overall size or the speed of growth of different traits. However, several studies suggest that they also entail immune costs to the offspring. In this study, we explored whether variation in yolk androgen concentration affected several measures of the constitutive and cell-mediated immune axes in the spotless starling (Sturnus unicolor). Using a within-brood design, we injected different doses of androgens (testosterone and androstenedione) into the eggs. Our study showed that experimentally increased yolk androgens led to sex-specific immunosuppression in both the innate and adaptive axes of the immune system. Both cell-mediated immune response (CMI) and lysozyme activity decreased with

*This paper was included by the Editor's request in a Focused Issue on "Early-life Effects on the Adult Phenotype: A Comparative Perspective."

†Corresponding author; e-mail: jaime.muriel.redondo@gmail.com, jaime.muriel @mncn.csic.es.

Physiological and Biochemical Zoology 90(1):106-117. 2017. (C) 2016 by The University of Chicago. All rights reserved. 1522-2152/2017/9001-5137\$15.00. DOI: $10.1086 / 688445$ increasing androgen levels injected into the egg in the case of male nestlings, whereas there were no effects on females. The effects that we found were always linear: no quadratic or threshold patterns were detected. We found no effects of the experimental treatment in hemolysis or agglutination capacity, but these measures were negatively correlated with CMI, suggesting negative correlation among different branches of the immune system. Blood (trypanosomes and hemosporidians) and intestinal (coccidia) parasites were not affected by the experimental increase of yolk androgen levels. Our results show that in our study species yolk androgens induce immunosuppression in some axes of the male nestling immune system. Further studies should analyze the proximate causes for these contrasting effects in different axes of the immune system and the reason for the differential impact on males and females.

Keywords: yolk hormones, testosterone, immunocompetence, parasites, androstenedione, maternal effects, Sturnus unicolor.

\section{Introduction}

Avian yolks contain important amounts of several androgens that are produced by female birds during ovulation (Groothuis et al. 2005b; Gil 2008). Yolk androgens have been shown to affect several aspects of embryo development and lead to both short- and long-term effects on various offspring traits (e.g., Strasser and Schwabl 2004; Partecke and Schwabl 2008). Although the effects vary among species, many studies suggest that during embryo and nestling development maternal androgens induce increases in overall size or the speed of growth of different traits (e.g., Pilz et al. 2004; Navara et al. 2006). These increases in growth can result in a modification of withinnest hierarchies among nestlings (Eising et al. 2001; Müller et al. 2004) or in overall differences between broods in growth and survival (Müller et al. 2007).

Given these apparent benefits, the occurrence of important levels of variation in androgen content within and among clutches suggests that androgen deposition may also entail costs for mothers or offspring (Gil et al. 1999). Several possibilities for constraints have been proposed, including mismatches between parental care and nestling demands (Hinde et al. 2010), negative effects on female fertility (Rutkowska et al. 2005), sexually antagonistic effects (Saino et al. 2006), and pleiotropic effects limiting optimal allocation for a given trait 
(Gil 2008). However, the hypothesis that has received the most attention in the literature so far is the immunocompetencehandicap hypothesis (Folstad and Karter 1992), which is based on the negative effects that androgens cause in the immune system. This hypothesis was initially proposed to account for possible costs limiting the development of exaggerated sexually selected traits. The evidence for the immunocompetencehandicap hypothesis is mixed, depending on the group of taxa that is examined (Roberts et al. 2004), but several studies suggest that immune costs, through either a direct or an indirect pathway, can play a role in the balance of costs and benefits of androgens (Owen-Ashley et al. 2004).

In the case of yolk androgens, several lines of evidence suggest that immune costs might limit the amount of androgens that bird eggs contain. For instance, cell-mediated and humoral immunity were found to be reduced in black-headed gull chicks (Larus ridibundus) that had been exposed to high levels of androgens in the egg (Groothuis et al. 2005a; Müller et al. 2005). A similar reduction in both branches of the immune system was found in jackdaws (Corvus monedula; Sandell et al. 2009). In the same line, experimentally infected house martins (Delichon urbica) and great tits (Parus major) laid eggs with lower androgen levels than control birds (Tschirren et al. 2004; Gil et al. 2006), although a recent study failed to find an effect after a similar experimental approach (Heylen et al. 2012). However, further studies in other species have found an enhanced immune response in nestlings hatched from androgentreated eggs (Navara et al. 2006) or no effects (e.g., Andersson et al. 2004; Tschirren et al. 2005; Rubolini et al. 2006a; Pitala et al. 2009) or else have uncovered roles for additional factors that affect this pattern. For instance, in zebra finches, Rutkowska et al. (2007) found that whereas experimental male nestlings suffered a reduction in $\mathrm{T}$-cell response, the pattern was inversed for female chicks. Also, in a previous study our team found that immune responses could actually be enhanced by yolk androgen injections, in this case in first broods of spotless starlings, presumably because of high resource availability (Muriel et al. 2015b).

Several studies have suggested that the trade-off between immunity and yolk androgens could arise by a variety of mechanisms, including the specific inhibition of immunity by androgens, based, for instance, on the presence of androgen receptors in some immune cells (Ahmadi and McCruden 2006; Gil and Culver 2011) or through the activation of a corticosteroid route to immune inhibition (Owen-Ashley et al. 2004). Regardless of the mechanism, a basic prediction is that there should be a trade-off between growth and immunity.

On the other hand, the immunological system is a set of defense mechanisms composed of several axes, including innate and adaptive immunity, and comprising cell-mediated and humoral responses. Therefore, it is not possible to measure the overall strength and efficiency of the immune system with a single immune assay (Adamo 2004). Previous studies have shown that correlations among immune responses need not be positive and that individuals may trade off different aspects of their immunocompetence (Salvante 2006; Forsman et al. 2008; Palacios et al.
2012). Trade-offs could be a consequence of an imbalance in the distribution of resources; several studies have shown that innate and adaptive immune responses may differ in costs (Lee et al. 2008; Palacios et al. 2009). Thus, any variation in maternally derived components, such as androgens, may mediate increased investment in one branch of immunity at the expense of another. For example, Clairardin et al. (2011) found that increased in ovo testosterone induced a trade-off between bactericidal activity and cutaneous immune response in house wrens (Troglodytes aedon). In a similar study conducted in zebra finches (Taeniopygia guttata), elevation of egg testosterone levels also resulted in long-lasting positive effects on humoral, but not cell-mediated, immune function (Tobler et al. 2010).

One problematic aspect in the study of yolk androgens is the variation in the injected doses in the different experiments conducted so far (Muriel et al. 2015a). This variation is likely to be a major cause of the diversity of effects found across studies, and it limits the general inferences that we can draw. Experimental dosage is an important issue, because steroids are known to present nonlinear, dose-response effects (Navara et al. 2005). We have previously shown (Muriel et al. 2015a) a variety of doseresponse patterns, depending on the morphological trait that is studied, in the spotless starling (Sturnus unicolor). In this study, we use a similar approach to inquire about the effects of an increasing amount of yolk androgens in a suite of immune responses and health status in the same species. We explore several components of the immune response, including cellmediated immune response (CMI), hematological traits, and several measures of constitutive immunity, including antibacterial capacity of plasma and lysozyme activity. Since immune deficiency is expected to lead to a reduction in the capacity of the organism to withstand attacks by parasites, we also analyze differences in the community of blood (trypanosome and hemosporidians) and intestinal (coccidia) parasites.

\section{Material and Methods}

\section{Study Species and Site}

The experiment was conducted in a large nest-box colony of spotless starlings (Sturnus unicolor) located near Madrid (Soto del Real). Yolk hormone manipulations were conducted between mid-April and mid-May 2010. The study area is a mixed woodland, used for cattle grazing, mostly composed of oak (Quercus pyrenaica) and ash (Fraxinus angustifolius). The spotless starling is a relatively long-lived, colonial, and sedentary passerine species that exhibits a facultatively polygynous breeding system (Moreno et al. 1999; Veiga 2002). This omnivorous songbird (Peris 1980) is sexually dimorphic, males being larger than females (Hiraldo and Herrera 1974). Incubation usually starts before the last egg is laid (3-6 eggs per clutch), and it is done mainly by females (for approximately $12 \mathrm{~d}$ ). Generally, females invest more than males in rearing the brood (Jimeno et al. 2014), although paternal care varies widely (Moreno et al. 1999). The nestling period lasts about 21-22 d (Cramp 1998). Females can lay up to two clutches per season, 
the first in early April and the second about the end of May in our study area (López-Rull et al. 2011). A replacement clutch could be laid as a result of the loss of the first clutch by predation or intraspecific competition (Müller et al. 2007; Muriel et al. $2015 a$ ). In this study, we used chicks belonging to first (from April 15 to May 19) and replacement broods (from May 9 to June 13). The daily average maximum temperature and precipitation (mean $\pm \mathrm{SE}$ ) recorded for each breeding attempt for the year of study were $16.82^{\circ} \pm 0.77^{\circ} \mathrm{C}$ and $2.35 \pm 0.84 \mathrm{~L} \mathrm{~m}^{-2}$ for the first brood and $21.07^{\circ} \pm 0.99^{\circ} \mathrm{C}$ and $1.79 \pm 0.60 \mathrm{~L} \mathrm{~m}^{-2}$ for replacement broods (data provided by the Spanish Meteorological Agency).

\section{Egg Injections}

From the end of March onward, nest boxes were inspected each day to determine laying date and order. Eggs were marked with a waterproof marker as they were laid. To minimize nest disturbance, injection of the first three eggs was carried out when the third egg was found in the nest, before incubation. All subsequent eggs were injected as they were laid, since incubation may already start with four eggs. Clutches were randomly assigned to one of three experimental treatments. Within each clutch, eggs were alternatively injected with either control or experimental injections, following laying order. The sequence of injections (i.e., starting with experimental or control) was modified between consecutive nests.

Experimental injections consisted of a combination of testosterone (T) and androstenedione (A4) dissolved in $10 \mu \mathrm{L}$ of sesame oil (ref. 85067, Sigma-Aldrich, Steinheim, Germany). We chose this combination of hormones because they appear together in the yolk (Schwabl 1993) and their concentrations are positively correlated (Groothuis and Schwabl 2002; Gil et al. 2004; Ruuskanen et al. 2009). Control eggs received an injection of sesame oil only $(10 \mu \mathrm{L})$. Experimental eggs received one of the following treatments: (1) low androgen dose: $12 \mathrm{ng} \mathrm{T}$ and $34 \mathrm{ng}$ A4 (refs. 86500 and A9630, respectively; Sigma-Aldrich); (2) intermediate androgen dose: $24 \mathrm{ng} \mathrm{T}$ and $68 \mathrm{ng}$ A4; or (3) high androgen dose: $48 \mathrm{ng} \mathrm{T}$ and $136 \mathrm{ng}$ A4. The low, intermediate, and high doses were equivalent to, respectively, 2, 4, and 8 SDs of the population means for an average 1.4-g egg ( $\mathrm{T}: 14 \mathrm{ng} /$ yolk [SD = 6.0], A4: $50 \mathrm{ng} /$ yolk [SD = 17.1]; Müller et al. 2007)). The maximum levels of yolk $\mathrm{T}$ and A4 that we have measured in this population are 36.3 and $198.4 \mathrm{ng} /$ yolk, respectively (Müller et al. 2007). The highdose injections that we used ( 8 SD) are slightly above and below, respectively, the maximum levels of T and A4 found in our population. Note, however, that previous studies indicate that injections in ovo do not necessarily mimic the natural distribution of hormones in the yolk, leading to variable exposure of the developing embryo (von Engelhardt et al. 2009). In addition, potential degradation or incomplete incorporation of the injected androgens into the yolk must be considered (Navara et al. 2005), as not all the hormone injected is finally assimilated by the developing embryo. In ovo injections were performed in the field with a standard U-50 insulin syringe (Terumo, Tokyo), following a standard protocol described elsewhere (Muriel et al. 2013, 2015a).

The experiment was carried out in 88 clutches, but we could use only 41 of them because of predation, destruction by other females, or impossibility of assigning hatchlings to their experimental group (this is not an unusual rate of nest failure in this population; Müller et al. 2007). We included in the analysis data from 153 chicks (75 males and 78 females), although sample sizes for the different tests differ because we were not able to collect enough blood from some individuals to perform all the immunological assays.

\section{Nestling Data and Sampling}

Broods were visited frequently around the predicted hatching date (10-11 d after the last egg was laid) to enable us to detect nestlings as they hatched and assign them to their specific experimental group. Chicks were labeled by subtle cuttings in their down.

Nestlings were measured on days 3, 6, 10, and 14 after hatching, in order to record growing patterns for a parallel study (Muriel et al. $2015 a$ ). In this analysis, we use data from day 14 , the moment of blood sampling, and the increase in mass from day 3 to day 14, which is referred to as "growth." Body mass was recorded with a digital balance (Ohaus Scout II SC2020, Shanghai, China; accuracy $=0.1 \mathrm{~g}$ ) and tarsus length with digital calipers (Mitutoyo Absolute, Kawasaki, Japan; accuracy $=0.01 \mathrm{~mm}$ ). All measurements were performed by the same person (J.M.), blindly to individual treatment whenever possible.

After measuring T-cell-mediated immune response (see below), we took a blood sample $(600 \mu \mathrm{L})$ of each nestling on day 15 after hatching. This sample was extracted from the jugular vein with heparinized syringes. Samples were kept on an ice box until arrival at the lab, and plasma was separated from the cell pellet by centrifuging at 5,000 $\mathrm{g}$ and $4^{\circ} \mathrm{C}$ for $10 \mathrm{~min}$. Plasma was stored in two separate aliquots at $-80^{\circ}$ until analysis. Also, fecal samples were collected from 142 of the 153 nestlings while they were handled; the samples were kept in ice until arrival at the lab and stored at $-80^{\circ}$ until analysis. In order to determine the presence of coccidian infections (Watve and Sukumar 1995), fecal samples were collected in the afternoon (1700-2100 hours), since previous studies have suggested that oocyst discharge is much greater in the afternoon than in the morning (Dolnik 1999; Brown et al. 2001).

\section{DNA Extraction and Molecular Sexing}

DNA extraction from blood samples was performed with an ammonium acetate method (Bensch and Akesson 2003). Sex determination was carried out by amplifying (through polymerase chain reaction [PCR]) an intron of the CHD1 genes on the avian sex chromosomes (Griffiths et al. 1998). PCR products were electrophoresed for $60-90 \mathrm{~min}$ at $100 \mathrm{~V}$ in $1.5 \%$ agarose gels stained with SYBR Safe (Invitrogen, Carlsbad, CA) and were visualized under ultraviolet light. In order to assure 
accurate assignment of the sex, the DNA extraction and PCR were carried out twice for 32 of the 153 samples. In all cases, the sex determination was identical.

\section{Cell-Mediated Immune Response}

We evaluated CMI on 14-d-old nestlings, using a dermal phytohemagglutinin (PHA) reaction in the wing web, following a standard protocol (Smits et al. 1999). After taking three measures of the thickness of a plucked area of the left wing web with a thickness gauge (Mitutoyo, Tokyo) to the nearest $0.01 \mathrm{~mm}$, we injected subcutaneously at that point $0.05 \mathrm{~mL}$ of a $5-\mathrm{mg} / \mathrm{mL}$ solution of PHA (L-8754, Sigma-Aldrich). After $24 \pm 1.3 \mathrm{~h}$, we took three new measurements of the thickness of the left wing web at the same point. As the repeatability of the measurements is high (Smits et al. 1999), we used the mean for statistical analysis. Cell-mediated immunocompetence was estimated as the difference between initial and final measurements of the left wing web swelling (Smits et al. 1999). All measurements were done by the same individual (J.M.), blindly with respect to treatment.

\section{Leukocyte and Hemoparasite Counts}

For identification of blood parasites and leucocytes, a drop of blood was smeared on one individually marked microscope slide. Once the blood had air-dried, we fixed the slide by $3 \mathrm{~min}$ of immersion in $100 \%$ methanol and stained it, using commercial Giemsa diluted with phosphate-buffered saline (PBS), pH 6.8 (1:2). Slides were examined under the microscope with the oil immersion objective $(\times 1,000$ magnification $)$ to estimate the proportions of different types of leucocytes and hemoparasites (Merino et al. 2001; Campbell and Ellis 2007). Estimates of the total white blood cell (WBC) count and intensity of infection were calculated per approximately 10,000 erythrocytes. Differential leukocyte counts were obtained by multiplying their proportions with respect to WBCs, which were classified as heterophils, eosinophils, basophils, lymphocytes, or monocytes. We also took the ratio of heterophils to lymphocytes $(\mathrm{H} / \mathrm{L})$ and the total leukocyte count as measures of physiological stress and immunity, respectively, in birds (Gross and Siegel 1983; Maxwell and Robertson 1998). By screening smears, we could identify intra- or extraerythrocytic hemoparasites only to the genus level. One person (J.M.) conducted all cell and parasite counts to eliminate variation between observers.

\section{Coccidia Abundance}

Protozoan coccidia are one of the most common intestinal parasites in birds (Zinke et al. 2004; Svobodova et al. 2015). Quantitative analysis of coccidian oocysts found in each fecal sample was carried out with a flotation technique (Villanúa et al. 2006). Fecal samples were extracted from collection tubes and extended in filter paper for $5 \mathrm{~min}$ to remove formaldehyde remnants. After that, approximately $0.5 \mathrm{~g}$ of feces was homog- enized and suspended in $5 \mathrm{~mL}$ of a saturated $\mathrm{ZnSO}_{4}$ solution (specific density: 1.18). Oocyst counts were performed with a MacMaster chamber, and calculation of their concentration (oocysts/g of feces) took into account the exact weight (to the nearest $0.001 \mathrm{~g}$ ) of each sample. On the basis of oocyst morphology (four sporozoites within each of two sporocysts), the coccidia detected were identified as Isospora spp., a protozoan gut parasite belonging to the Eimeria complex. Because of its direct life cycle, avian Isospora species require no vector for the spread of infection, and transmission occurs if an appropriate host ingests sporulated oocysts (Fayer and Reid 1982). We did not detect other intestinal parasite propagules in the samples analyzed.

\section{Hemolysis-Hemagglutination Assays}

Levels of natural antibodies and complement were quantified following Matson et al. (2005). We prepared two-fold serial dilutions of plasma into PBS across a 96-well plate, using one row per bird (12 wells). Each row contained plasma at dilutions ranging from 1 through $1: 1.024$ in volumes of $25 \mu \mathrm{L}$. We added to each well $25 \mu \mathrm{L}$ of a $0.1 \%$ sheep red blood cell (SRBC) suspension prepared with blood extracted from a sheep housed at the Dehesa de Galiana farm (Ciudad Real, Spain) less than $48 \mathrm{~h}$ before the assay and stored refrigerated until processing. Plates were covered and incubated at $37^{\circ} \mathrm{C}$ for $60 \mathrm{~min}$, tilted at a $45^{\circ}$ angle for an additional $60 \mathrm{~min}$, and then scanned under a stereomicroscope. We scored each sample by taking the value of the lowest plasma dilution that was sufficient to induce hemagglutination and hemolysis. Therefore, higher scores reflect, respectively, higher levels of natural antibodies and higher combined activity of the complement with natural antibodies. All scores were performed by the same person (M.E.O.-S.), who was blind to the identity of each sample. Repeatabilities, for a subset of 31 random samples assayed in duplicate, were high for both hemolysis $(r=0.86$, $\left.F_{30,31}=13.8, P<0.001\right)$ and hemagglutination $\left(r=0.91, F_{30,31}=\right.$ 20.1, $P<0.001)$.

\section{Lysozyme Activity}

Lysozyme is one the main antimicrobial proteins of the blood, being produced by most types of leukocytes (Gill 1995). To measure lysozyme activity of plasma samples, we used a 600-mg/L suspension of Micrococcus lysodeikticus (ref. M3770$5 \mathrm{G}$, Sigma-Aldrich) in PBS. In each well of a 96-well plate, we added $200 \mu \mathrm{L}$ of this bacterial suspension to $20-\mu \mathrm{L}$ plasma or PBS wells, which were used as blanks. The activity of lysozyme is proportional to the rate of absorbance reduction because of the lysis M. lysodeikticus present in the suspension. To quantify this process, plates were incubated at $37^{\circ} \mathrm{C}$, and we measured absorbance at $850 \mathrm{~nm}$ at 15, 30, 45 and $60 \mathrm{~min}$, using a microplate reader (Biotek Powerwave XS2, Winooski, VT). Plasma samples were assayed in duplicate. For each sample, we calculated the regression slope between absorbance (after subtracting the absorbance of blanks) and time. In order to quantify lysozyme con- 
centrations (in $\mu \mathrm{g} / \mathrm{mL}$ ), a standard curve elaborated by serially diluting crystallized lysozyme (ref. L-6876, Sigma-Aldrich) in PBS was also included in all plates and treated in the same way as plasma samples. The repeatability of lysozyme quantification was high $\left(r=0.90, F_{48,49}=19.9, P<0.001\right)$.

\section{Statistical Analysis}

All calculations were performed in the R language, version 3.1.0 (R Development Core Team 2015). We analyzed the data, applying general lineal mixed models with the lmer function in the "lme4" package (Bates et al. 2014), and, depending on the data distribution, we used Gaussian, Poisson, or binomial negative distributions. Box-cox transformations were performed on the raw data and followed the formula $x^{\prime}=\left(x^{\lambda}-1\right) / \lambda$, where $\lambda$ was specific to each measurement as follows: swelling response to PHA: 0.56 ; body mass at $14 \mathrm{~d}$ of age: 3.87 ; lysis levels: 0.86 ; agglutination: 1.15; growth: 3.14 ; lysozyme activity: $-1.16 ; \mathrm{H} / \mathrm{L}$ : -0.29 ; eosinophils/total leukocytes: 0.40 ; and total leucocytes/ erythrocytes: 0.25 . The remainder of measurements did not require transformation. Brood (i.e., first vs. replacement), growth, experimental treatment, and sex were entered as fixed factors (predictor variables). Treatment was treated as continuous rather than categorical because androgen doses increased stepwise (Muriel et al. 2015a) and because we explicitly wanted to test whether effects conformed to a linear or a quadratic relationship (Navara et al. 2005; Muriel et al. 2015a). In all cases, nest was defined as a random effect affecting the model intercept. Since treatment may influence hatching speed and body mass (Muriel et al. 2015a), we also included embryonic developmental period (days to hatch) and body weight at $14 \mathrm{~d}$ of age as fixed factors in the initial models. All biologically meaningful double interactions were included in the original model. Information criteria (lowest Akaike information criterion value) were used to select the final models.

\section{Results}

\section{Cell-Mediated Immune Response}

The best-supported model to explain the swelling response to PHA showed an interaction between treatment and sex, indicating that immune response was differentially affected by treatment in males and females (table 1). The quadratic effect of treatment was not retained in the model. The significant interaction resulted from negative and positive trends for males and females, respectively (fig. 1). However, separate linear mixed models for males and females failed to find a significant pattern of treatment in either sex (males: $\chi^{2}=2.07, P=0.15$, estimate [SE] $=-0.045[0.03]$; females: $\chi^{2}=2.86 ; P=0.09$, estimate $[\mathrm{SE}]=0.051[0.03]$ ). Furthermore, after body size was controlled for, birds that grew more during their nestling period also had stronger immune responses (fig. 2). Embryonic developmental period did not affect swelling response. Irrespective of treatment, CMI showed a seasonal decline in replacement broods and lower responses in females (table 1).
Table 1: Results of the linear mixed model testing the predictors of nestling cell-mediated immune response (swelling response), where nest is declared as random effect

\begin{tabular}{lrrr}
\hline Term & $\chi^{2}$ & $P$ & Estimate (SE) \\
\hline Treatment & 1.01 & .317 & $-.031(.03)$ \\
Sex & 4.54 & .033 & $-.375(.17)$ \\
Preswelling & .34 & .555 & $.046(.08)$ \\
Brood & 4.30 & .038 & $-.393(.19)$ \\
Body mass & 2.10 & .147 & $-.245(.17)$ \\
Growth & 7.42 & .006 & $.497(.17)$ \\
Treatment $\times$ sex & 4.91 & .027 & $.095(.04)$ \\
\hline
\end{tabular}

Note. $\chi^{2}(\mathrm{df}=1)$ and $P$ values arise from a type III analysis of deviance on the model. In the case of factors, estimates refer to the second level of each group (females in the case of sex, and replacement broods in the case of brood). Sample size is 153 individuals.

\section{Constitutive Immunity Measurements}

Hemolysis and Agglutination. Lysis levels were not affected by treatment, sex, body mass, embryonic developmental period, or growth (all $P>0.23$ ), but they strongly increased in replacement broods relative to first broods $\left(\chi^{2}=12.32, P<0.001\right.$; estimate [SE] for the replacement brood: 0.72 [0.20]). Agglutination was similarly dependent on brood $\left(\chi^{2}=9.18, P<0.01\right.$; estimate [SE] for the replacement brood: $0.65[0.21])$, increased with increasing body mass $\left(\chi^{2}=6.32, P<0.02\right.$; estimate [SE]: 0.24 $[0.09])$, and was not affected by treatment, sex, embryonic developmental period, or growth $(P>0.18)$. The quadratic effect of treatment was not retained in the model.

Lysozyme Activity in Plasma. The GLM for lysozyme activity showed a significant interaction between treatment and sex, with males displaying lower levels of lysozyme activity with increasing yolk androgen dose and females displaying no effect (table 2; fig. 3), and neither brood, body mass, embryonic developmental period, nor growth was significant. Separate models for males and females confirmed this interaction, showing a highly significant negative effect of treatment on males $\left(\chi^{2}=6.56, P<0.01\right.$, estimate $\left.[\mathrm{SE}]:-0.09[0.03]\right)$ and a nonsignificant positive trend for females $\left(\chi^{2}=2.24, P=0.14\right.$, estimate [SE]: 0.10 [0.069]). The quadratic effect of treatment was not retained in any of the models.

\section{Correlations among Immunological Measurements}

We ran pairwise mixed models on the normalized scores of the four measurements of immunity that we obtained, controlling for nest as random factor (table 3). The data for agglutination and hemolysis scores show a very strong relationship among them. Cell-mediated immunity is, however, negatively related to agglutination and shows a similar trend with respect to hemolysis. Lysozyme activity stands out as an independent component of the immune system, with very weak relationships with the rest (table 3). 


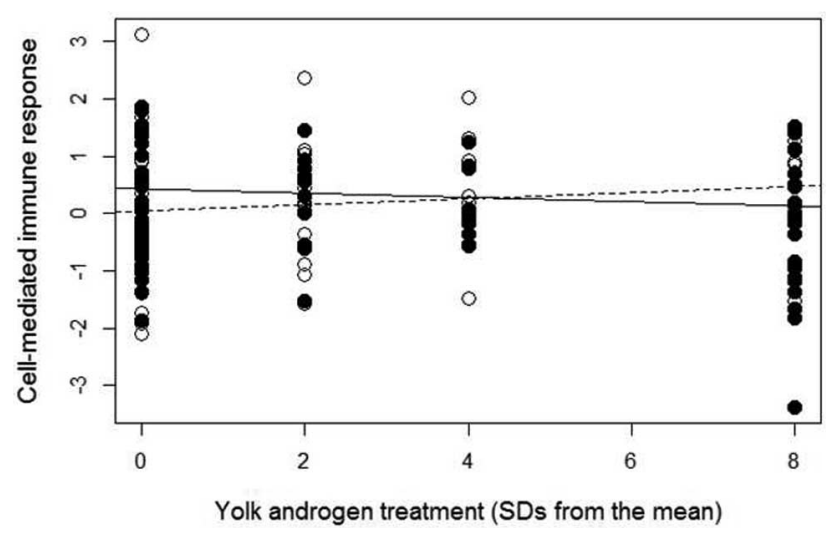

Figure 1. Effects of yolk androgen treatment on cell-mediated immune response (swelling of the wing web in response to phytohemagglutinin injection). Filled circles and the solid line represent males, and open circles and the dotted line represent females.

\section{Hematological Traits}

We ran individual models for percentages of each leukocyte type over total leukocytes, for total number of leukocytes, and for $\mathrm{H} / \mathrm{L}$ (table 4), testing the predictors and respecting the specific error distribution of each variable. Treatment was not related to any trait, although there was a nonsignificant tendency $(P=0.07)$ for basophils to decrease with increasing yolk androgen dose. There were no significant differences between sexes in the composition of blood cell types. Eosinophils increased with body mass, whereas H/L decreased. Replacement broods were characterized by significantly lower levels of basophils and monocytes and a reduction in H/L.

\section{Parasites}

We detected no malaria parasites (Haemoproteus, Plasmodium, or Leucocytozoon) in the first 50 blood smears that we analyzed, and so we stopped checking for them. However, Trypanosoma spp. were found, with a moderate prevalence $(19.73 \%, N=152)$. A binomial GLM showed that the presence of Trypanosoma in blood was not related to treatment, sex, or body mass (for all estimates, $P>0.37$ ) but strongly increased from first to replacement broods $(F=11.23, P<0.001$; estimate [SE] for the replacement brood: 2.59 [0.77]). Using a generalized linear mixed model with a negative binomial distribution, we found that the number of coccidia in feces was also not related to treatment, sex, or body mass (all $P>0.10$ ) but strongly increased from first to replacement broods $\left(\chi^{2}=9.57, P<0.01\right.$; estimate [SE] for the replacement brood: $1.64[0.51])$.

\section{Discussion}

Our study examined how several aspects of nestling innate immunity were modified as a response to an experimental increase in yolk androgen levels. The general pattern indicates that the two sexes react differently in this species, males being more immunologically compromised by yolk androgens than females (see below). There was no general effect across sexes, even though our treatment encompassed a wide range of yolk androgen doses and was designed to test for possible dose-dependent effects. There were sex-specific patterns for CMI and lysozyme activity, but neither innate lysis, agglutination efficiency, nor general immune condition as measured by hematological traits showed immune suppression. In agreement with these patterns, neither hematozoan parasites nor coccidial infection was increased as a result of the yolk androgen manipulation.

We detected two sex-specific patterns, in which both male plasma lysozyme activity and male CMI decreased with increasing yolk androgen levels. These results are consistent with a previous study conducted in the same starling population, where male chicks that hatched from yolk androgen-treated eggs suffered a slight suppression of their cell-mediated immune responsiveness (Muriel et al. 2013). A similar pattern has been previously found in the zebra finch Taenyiopigya guttata (Rutkowska et al. 2007), which matches with the sex-specific effects of yolk androgens on growth in that species (von Engelhardt et al. 2006).

Evidence from previous studies shows that yolk androgen manipulations can affect the growth of males and females differently in some species (Saino et al. 2006; von Engelhardt et al. 2006), and a recent review concluded that these differential effects tend to happen more often in species with strong sexual size dimorphism (Tschirren 2015). In a parallel study using the same experimental setup employed here, we found no sexual differences in the effect of elevated yolk androgen resulting in somatic growth (Muriel et al. 2015a). Therefore, our data suggest that this differential effect of sex is independent of absolute size and is rooted in sex-specific processes, so that only males, the larger sex, pay an extra immunological cost, as previously shown in several studies across a diverse range of species (Fargallo et al. 2002; Müller et al. 2003; Lobato et al. 2008). Since male and female chicks in our study species have similar levels of T (Müller et al. 2007; Gil et al. 2008), the higher immune susceptibility of the former should arise by differences

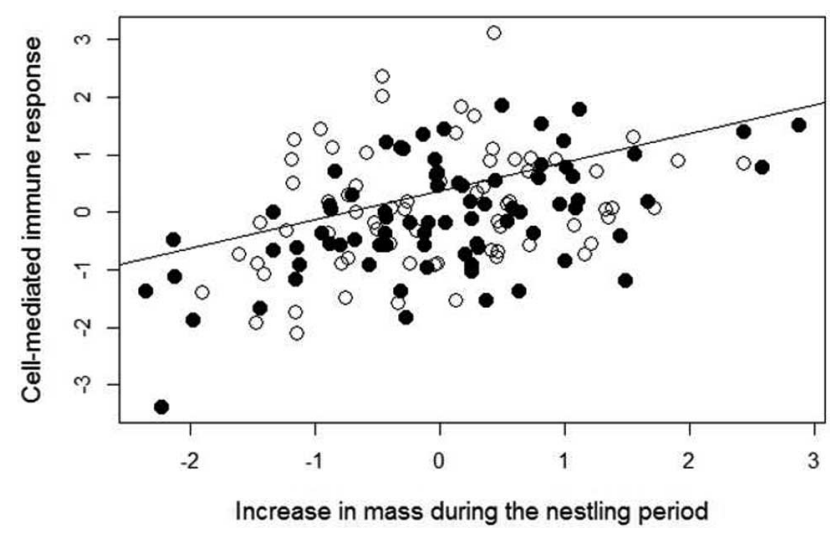

Figure 2. Relationship between cell-mediated immune response (normalized swelling of the wing web in response to phytohemagglutinin injection) and growth during the nestling stage. Filled circles represent males and open circles females. 
Table 2: Results of a linear mixed model testing the predictors of nestling lysozyme activity, where nest is declared as random effect

\begin{tabular}{lrcc}
\hline Term & $\chi^{2}$ & $P$ & Estimate $(\mathrm{SE})$ \\
\hline Treatment & 3.17 & .074 & $-.089(.05)$ \\
Sex & .76 & .381 & $-.234(.27)$ \\
Brood & 2.63 & .104 & $-.346(.21)$ \\
Body mass & .78 & .375 & $-.185(.21)$ \\
Growth & .04 & .833 & $-.044(.21)$ \\
Treatment $\times$ sex & 6.29 & .012 & $.182(.07)$ \\
\hline
\end{tabular}

Note. $\chi^{2}(\mathrm{df}=1)$ and $P$ values arise from a type III analysis of deviance on the model. In the case of factors, estimates refer to the second level of each group (females in the case of sex, and replacement broods in the case of brood). Sample size is 93 individuals.

in hormone metabolism or function (Møller et al. 1998; Duffy et al. 2000; Moreno et al. 2001; Fargallo et al. 2002).

Most experimental injections of yolk androgens typically use a single dose, and the amount that is injected varies widely between studies (Muriel et al. 2015a). Avian studies have rarely examined dose-response patterns in relation to immunological costs. However, one study has found that high and low doses of T have opposed effects on cell-mediated immunity in nestlings (Navara et al. 2005). In the present study, we found that the effects of androgens on immunity were lineal, increasing with increasing dose, and that no quadratic effects were retained in the models. This is in contrast to growth patterns in morphological traits, which in a parallel study of these birds were found to show a diversity of linear and nonlinear patterns in relation to dose (Muriel et al. 2015a). This can be taken to suggest that the immunological costs that we detected increase monotonically within the doses that we used and that growth patterns result from a compromise between enhanced somatic growth and immune costs. Thus, the contrasted dose-response patterns result from the former being adaptively modulated, whereas the latter are not controlled by the individual. Indeed, theory predicts that benefits derived from high androgen levels are counteracted by negative effects on immunity (Folstad and Karter 1992). In the case of yolk androgen levels, several studies have found support for this hypothesis, in both the cellular and humoral axes of immunity (Müller et al. 2005; Groothuis et al. 2008; Sandell et al. 2009). However, other studies have found no effects (Rubolini et al. 2006b) or conflicting evidence. For instance, in another experiment in the same species, we found that differences in lymphocyte proliferation between treatment and control nestlings varied, depending on the environmental conditions when the study was done (Muriel et al. 2015b). Although our results do not show an interaction between treatment and brood, there may be a broader environmental effect in terms of breeding season. Since differences in environmental conditions during early development may differentially affect male and female offspring (Blondel et al. 2002), it is possible that the adverse weather conditions experienced during first and replacement broods in 2010, cooler and drier than what is usually experienced during this period (Muriel et al. 2015b), entailed higher immunological costs, especially in androgentreated males.

Although we found no evidence of any direct statistical correlation between CMI and lysozyme activity in plasma (but see Clairardin et al. 2011), both measurements showed the same sex-specific responses to high yolk androgen levels. These common results in males could be interpreted on the light of common components in the innate and adaptive axes of the immune system (Forsman et al. 2010; Vinkler et al. 2010). In fact, some studies have shown that the PHA swelling response is an integration of both specific and induced immunity and also of constitutive and nonspecific immunity (Martin et al. 2006a; Salaberria et al. 2013). However, contrary to lysozyme activity, CMI was also influenced by several variables. Thus, we found that CMI was positively correlated with nestling growth, which is consistent with previous studies finding a less developed CMI in nestlings with lower growth rates (Hõrak et al. 1999, 2000). Since the activation of an immune response has energetic and/or nutrient costs that may interfere with metabolic processes (Demas et al. 1997), the positive relationship between cell-mediated response and the increase in mass that we found suggests that birds did not trade off one against the other.

We found that CMI, as well as several hematological traits, such as basophils and monocytes, decreased as the breeding season advanced, as is often the case in other studies (Sorci et al. 1997; López-Rull et al. 2011). We have previously shown a negative effect of clutch laying date on nestling body condition in the same study population (Muriel et al. 2015a). This pattern is in agreement with the lower immune responses that we detected in this study. This pattern could arise because of lower parental quality, since high-quality birds breed earlier in the season and are probably less likely to lose their first-laid clutches (Brinkhof et al. 1999; Verhulst and Nilsson 2008). In addition, differences can arise because of differences in overall food quantity and quality or differential exposure to parasites and pathogens (Sorci et al. 1997; Biard et al. 2015; Muriel et al. 2015b). In agreement with this possibility, we found an

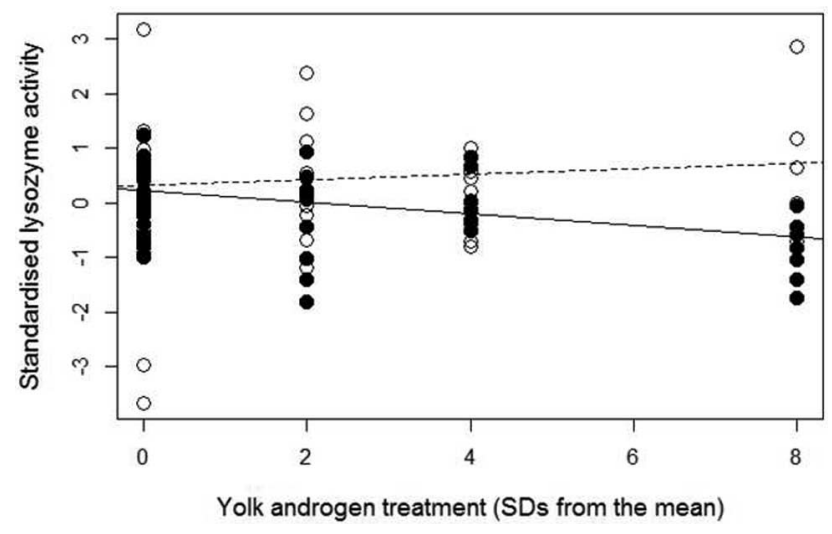

Figure 3. Effects of yolk androgen treatment on lysozyme antibacterial activity. Filled circles and the solid line represent males, and open circles and the dotted line represent females. 
Table 3: Correlation estimates (SE) among the four immunological measurements obtained

\begin{tabular}{lccc}
\hline & Hemolysis & Agglutination & $\begin{array}{c}\text { Lysozyme } \\
\text { activity }\end{array}$ \\
\hline CMI & $-.181(.10)$ & $-.245(.09)^{\star}$ & $-.012(.09)$ \\
Hemolysis & & $.791(.07)^{\star * *}$ & $-.004(.09)$ \\
Agglutination & & & $-.019(.09)$ \\
\hline
\end{tabular}

Note. Data obtained from linear mixed models that include only pairwise terms, controlling for nest as random factor. Sample size is 94 individuals. $\mathrm{CMI}=$ cell-mediated immune response.

${ }^{*} P<0.05$.

${ }^{* * *} P<0.001$

increase of trypanosomes and coccidia in replacement broods compared to first broods.

In a previous study, we used an in vitro assay to study T-cell proliferation patterns in nestlings after yolk androgen manipulation and found that it varied, depending on the season (Muriel et al. 2015b). It is not possible to directly compare those patterns with those reported here because here we have used a measure of the external swelling response, which involves not only proliferation of $\mathrm{T}$-cells but also the secretion of proin- flammatory cytokines that recruit and activate effector cells and phagocytes such as basophils, heterophils, and macrophages (Martin et al. 2006a; Salaberria et al. 2013) and thus involves both innate and adaptive components of the immune system (Stadecker et al. 1977; Bílková et al. 2015).

The different components of the immune system do not always respond in the same way, and studies show that they can interact and trade off with one another (Martin et al. 2006b; Palacios et al. 2007; Forsman et al. 2008). We found that hemolysis and agglutination (measures of natural antibody levels and complement activation, respectively; Matson et al. 2005) covaried positively, as found before in other avian species (Matson et al. 2006). This correlation is expected because natural antibodies, responsible for hemagglutination, are also involved in the hemolysis process, as antibodies interact with the antigen (SRBC) to create complexes on which complement proteins responsible for lysis act (Matson et al. 2005). However, these two measures covaried negatively with cell immunity, showing, perhaps, different immune strategies between individuals. It is possible that differences in maternal or environmental factors, such as female quality or weather conditions, could explain this pattern, since these two groups of immune responses showed opposite patterns in relation to breeding event (e.g., first vs. replacement clutches). Lysozyme activity, however,

Table 4: Results of models testing the predictors of several hematological traits, where nest is declared as random effect

\begin{tabular}{lccc}
\hline White blood cell type, terms & $\chi^{2}$ & Estimate (SE) & $P$ \\
\hline Heterophil/lymphocyte ratio: & & & \\
$\quad$ Treatment & 1.41 & $.03(.02)$ & .234 \\
Sex & .01 & $-.01(.15)$ & .975 \\
Brood & 8.64 & $-.54(.18)$ & .003 \\
$\quad$ Body mass & 9.30 & $-.27(.08)$ & .002 \\
Eosinophils/total leukocytes (\%): & & & \\
$\quad$ Treatment & .20 & $.01(.02)$ & .653 \\
Sex & .05 & $.04(.15)$ & .809 \\
Brood & 1.70 & $.26(.19)$ & .192 \\
Body mass & 6.60 & $.24(.09)$ & .010 \\
Basophils/total leukocytes (\%): & & & \\
Treatment & 3.24 & $-.03(.02)$ & .071 \\
Sex & .87 & $-.12(.12)$ & .350 \\
Brood & 45.2 & $-.90(.13)$ & $<.001$ \\
Body mass & .47 & $-.05(.07)$ & .488 \\
Monocytes/total leukocytes (\%): & & & \\
Treatment & .01 & $-.01(.02)$ & .989 \\
Sex & 1.03 & $-.15(.15)$ & .310 \\
Brood & 36.75 & $-.97(.16)$ & $<.001$ \\
Body mass & .89 & $-.08(.08)$ & .345 \\
Total leucocytes/erythrocytes (\%): & & & \\
Treatment & 2.42 & $-.04(.03)$ & .119 \\
Sex & 3.10 & $.28(.16)$ & .078 \\
Brood & .03 & $.03(.19)$ & .853 \\
Body mass & .13 & $.10(.09)$ & .256 \\
\hline Note & & & \\
\hline
\end{tabular}

Note. Models are either linear mixed models on normalized values, when possible, or generalized linear mixed models with Poisson error distributions. $\chi^{2}(\mathrm{df}=1)$ and $P$ values arise from a type III analysis of deviance on the model. In the case of factors, estimates refer to the second level of each group (females in the case of sex and replacement broods in the case of brood). Sample size is 153 individuals. 
behaved independently from any other component of the immune system included in this study. This is not the first time that immunocompetence estimates have been found to differ, showing negative correlation. For instance, it has been found in house wrens that humoral and cell-mediated responses covary negatively among broods, suggesting a trade-off in immunity (Forsman et al. 2008). In fact, a study in that same species has shown that experimentally increased in ovo $\mathrm{T}$ induces a trade-off between bactericidal activity and cutaneous immune response (Clairardin et al. 2011). Thus, our results show that immunosuppression resulting from high yolk androgen levels affected components of both the innate and adaptive axes of the immune system in males. Yolk androgens may prime the development and expression of various components of the immune system, such as cell-mediated and humoral immune function, differently (Sandell et al. 2009), with sex- and context-specific consequences (Pigeon et al. 2013).

Immunosuppression due to high androgen levels could make individuals more susceptible to disease. However, we found no evidence for malaria infection in any of the individuals during the nestling phase. This could be due to blood samples being taken too early in development (Merino and Potti 1995; Cosgrove et al. 2006). On the other hand, we found only a few chicks infected with trypanosomes, species that have probably a shorter prepatent period (Merino and Potti 1995). In contrast, the prevalence of infection by coccidia (Isospora spp.) was high, although there were no differences in relation to yolk androgen levels. In line with our results, Tschirren et al. (2005) found no indication that high concentrations of yolk $\mathrm{T}$ increase the nestling's susceptibility to ectoparasites. Chicks in replacement broods had higher levels of both coccidia and trypanosomes. This pattern is likely due to differences in abiotic factors, such as temperature or humidity (Brooker et al. 2006; Svobodova et al. 2015), or to seasonal differences in diet that would imply different exposure to parasites (Dolnik et al. 2010).

To sum up, our study showed that experimentally increased yolk androgens led to sex-specific immunosuppression of two measures of innate and adaptive axes of the immune system. Despite this, there was no evidence of increased susceptibility to blood or intestinal parasites. We found a negative correlation among different branches of the immune system, suggesting that future studies about the effects of maternal androgens on immune response should consider a wider range of immunological assays that cover as many branches of the innate and adaptive immune system as possible. In addition, we note the importance of exploring the sex- and context-specific effects of yolk androgens to understand more precisely the role of maternal effects in the offspring immune response.

\section{Acknowledgments}

This study was financed by project grants CGL2008-03501 and CGL2011-26318 to D.G. (Ministerio de Ciencia e Innovación [MICINN]). J.M. was supported by an FPI (Formación de Personal Investigador) grant (BES-2009-021383) from MICINN.
L.P.-R. was supported by a postdoctoral grant from MICINN through the Juan de la Cierva Subprogram (JCI-2008-2059), followed by a postdoctoral contract from the Spanish Ministry of Economy and Competitiveness through the Severo Ochoa Program for Centres of Excellence in Research, Development, and Innovation (SEV-2012-0262). M.E.O.-S. was supported by a postdoctoral grant from MICINN through the Juan de la Cierva Subprogram (JCI-2009-03865), followed by a Marie Curie IntraEuropean Fellowship of the European Union's 7th Framework Program (2012-328328). Permission to work in the study area was granted by the Ayuntamiento de Soto del Real. Capture and manipulation of birds were authorized by the Consejería de Medio Ambiente (Comunidad de Madrid). The authors declare no conflict of interest. Raquel Monclús advised us on statistics.

\section{Literature Cited}

Adamo S.A. 2004. How should behavioural ecologists interpret measurements of immunity? Anim Behav 68:14431449.

Ahmadi K. and A.B. McCruden. 2006. Macrophage may responses to androgen via its receptor. Med Sci Monit 12: BR15-BR20.

Andersson S., T. Uller, M. Lohmus, and F. Sundstrom. 2004. Effects of egg yolk testosterone on growth and immunity in a precocial bird. J Evol Biol 17:501-505.

Bates D., M. Maechler, B. Bolker, and S. Walker. 2014. lme4: linear mixed-effects models using "Eigen" and S4. R package version 1.1-6. http://CRAN.R-project.org/package $=$ lme4.

Bensch S. and S. Akesson. 2003. Temporal and spatial variation of hematozoans in Scandinavian willow warblers. J Parasitol 89:388-391.

Biard C., K. Monceau, S. Motreuil, and J. Moreau. 2015. Interpreting immunological indices: the importance of taking parasite community into account. An example in blackbirds Turdus merula. Methods Ecol Evol 6:960-972.

Bílková B., J. Vinklerová, and M. Vinkler. 2015. The relationship between health and cell-mediated immunity measured in ecology: phytohaemagglutinin skin-swelling test mirrors blood cellular composition. J Exp Zool A 323:767-777.

Blondel J., P. Perret, M.-C. Anstett, and C. Thébaud. 2002. Evolution of sexual size dimorphism in birds: test of hypotheses using blue tits in contrasted Mediterranean habitats. J Evol Biol 15:440-450.

Brinkhof M.W.G., P. Heeb, M. Kolliker, and H. Richner. 1999. Immunocompetence of nestling great tits in relation to rearing environment and parentage. Proc R Soc B 266:23152322.

Brooker S., A.C.A. Clements, and D.A.P. Bundy. 2006. Global epidemiology, ecology and control of soil-transmitted helminth infections. Pp. 221-261 in S.I. Hay, A. Graham, and D.J. Rogers, eds. Global mapping of infectious diseases: methods, examples and emerging applications. Advances in Parasitology, Vol. 62. Academic Press, London. 
Brown M.A., S.J. Ball, and D. Holman. 2001. The periodicity of isosporan oocyst discharge in the greenfinch (Carduelis chloris). J Nat Hist 35:945-948.

Campbell T.W. and C.K. Ellis. 2007. Avian and exotic animal hematology and cytology. Wiley-Blackwell, Oxford.

Clairardin S.G., C.A. Barnett, S.K. Sakaluk, and C.F. Thompson. 2011. Experimentally increased in ovo testosterone leads to increased plasma bactericidal activity and decreased cutaneous immune response in nestling house wrens. J Exp Biol 214: 2778-2782.

Cosgrove C.L., S.C.L. Knowles, K.P. Day, and B.C. Sheldon. 2006. No evidence for avian malaria infection during the nestling phase in a passerine bird. J Parasitol 92:1302-1304.

Cramp S. 1998. The complete birds of the western Palaearctic. Oxford University Press and OptiMedia, Oxford. CDROM.

Demas G.E., V. Chefer, M.I. Talan, and R.J. Nelson. 1997. Metabolic costs of mounting an antigen-stimulated immune response in adult and aged C57BL/6J mice. Am J Physiol Regul Integr Comp Physiol 273:R1631-R1637.

Dolnik O.V. 1999. Diurnal oocyst periodicity in Isospora dilatata (Sporozoa, Eimeriidae) from the common starling (Sturnus vulgaris) in nature. Parazitologiya (St Petersbg) 33: 74-80.

Dolnik O.V., V.R. Dolnik, and F. Bairlein. 2010. The effect of host foraging ecology on the prevalence and intensity of coccidian infection in wild passerine birds. Ardea 98:97103.

Duffy D.L., G.E. Bentley, D.L. Drazen, and G.F. Ball. 2000. Effects of testosterone on cell-mediated and humoral immunity in non-breeding adult European starlings. Behav Ecol 11:654-662.

Eising C.M., C. Eikenaar, H. Schwabl, and T.G.G. Groothuis. 2001. Maternal androgens in black-headed gull (Larus ridibundus) eggs: consequences for chick development. Proc R Soc B 268:839-846.

Fargallo J.A., T. Laaksonen, V. Poyri, and E. Korpimäki. 2002. Inter-sexual differences in the immune response of Eurasian kestrel nestlings under food shortage. Ecol Lett 5:95101.

Fayer R. and W.M. Reid. 1982. Control of coccidiosis. Pp. 453487 in P.L. Long, ed. The biology of the coccidia. University Park, Baltimore.

Folstad I. and A.J. Karter. 1992. Parasites, bright males, and the immunocompetence handicap. Am Nat 139:603-622.

Forsman A.M., S.K. Sakaluk, C.F. Thompson, and L.A. Vogel. 2010. Cutaneous immune activity, but not innate immune responsiveness, covaries with mass and environment in nestling house wrens (Troglodytes aedon). Physiol Biochem Zool 83:512-518.

Forsman A.M., L.A. Vogel, S.K. Sakaluk, J.L. Grindstaff, and C.F. Thompson. 2008. Immune-challenged house wren broods differ in the relative strengths of their responses among different axes of the immune system. J Evol Biol 21:873-878.

Gil D. 2008. Hormones in bird eggs: physiology, ecology and behavior. Adv Study Behav 38:337-398.
Gil D., E. Bulmer, P. Celis, and M. Puerta. 2008. Increased sibling competition does not increase testosterone or corticosterone levels in nestlings of the spotless starling (Sturnus unicolor). Horm Behav 54:238-243.

Gil D. and R. Culver. 2011. Male ornament size in a passerine predicts the inhibitory effect of testosterone on macrophage phagocytosis. Funct Ecol 25:1278-1283.

Gil D., J.A. Graves, N. Hazon, and A. Wells. 1999. Male attractiveness and differential testosterone investment in zebra finch eggs. Science 286:126-128.

Gil D., G. Leboucher, A. Lacroix, R. Cue, and M. Kreutzer. 2004. Female canaries produce eggs with greater amounts of testosterone when exposed to preferred male song. Horm Behav 45:64-70.

Gil D., A. Marzal, F. De Lope, M. Puerta, and A.P. Møller. 2006. Female house martins (Delichon urbica) reduce egg androgen deposition in response to a challenge of their immune system. Behav Ecol Sociobiol 60:96-100.

Gill J. 1995. Serum lysozyme level in the European bison, Bison bonasus (L). Comp Biochem Physiol B 110:235-240.

Griffiths R., M.C. Double, K. Orr, and R.J.G. Dawson. 1998. A DNA test to sex most birds. Mol Ecol 7:1071-1075.

Groothuis T.G.G., C. Carere, J. Lipar, P.J. Drent, and H. Schwabl. 2008. Selection on personality in a songbird affects maternal hormone levels tuned to its effect on timing of reproduction. Biol Lett 4:465-467.

Groothuis T.G.G., C.M. Eising, C. Dijkstra, and W. Müller. 2005a. Balancing between costs and benefits of maternal hormone deposition in avian eggs. Biol Lett 1:78-81.

Groothuis T.G.G., W. Müller, N. von Engelhardt, C. Carere, and C. Eising. 2005b. Maternal hormones as a tool to adjust offspring phenotype in avian species. Neurosci Biobehav Rev 29:329-352.

Groothuis T.G.G. and H. Schwabl. 2002. Determinants of within- and among-clutch variation in levels of maternal hormones in black-headed gull eggs. Funct Ecol 16:281289.

Gross W.B. and H.S. Siegel. 1983. Evaluation of the heterophil lymphocyte ratio as a measure of stress in chickens. Avian Dis 27:972-979.

Heylen D., W. Müller, T.G.G. Groothuis, and E. Matthysen. 2012. Female great tits do not alter their yolk androgen deposition when infested with a low-transmittable ectoparasite. Behav Ecol Sociobiol 66:287-293.

Hinde C.A., R.A. Johnstone, and R.M. Kilner. 2010. Parentoffspring conflict and coadaptation. Science 327:13731376.

Hiraldo F. and C.M. Herrera. 1974. Dimorfismo sexual y diferenciación de edades en Sturnus unicolor Temm. Doñana Acta Vertebr 1:149-170.

Hõrak P., I. Ots, L. Tegelmann, and A.P. Møller. 2000. Health impact of phytohaemagglutinin-induced immune challenge on great tit (Parus major) nestlings. Can J Zool 78:905-910.

Hõrak P., L. Tegelmann, I. Ots, and A.P. Møller. 1999. Immune function and survival of great tit nestlings in relation to growth conditions. Oecologia 121:316-322. 
Jimeno B., J. Muriel, L. Pérez-Rodríguez, and D. Gil. 2014. Sexual differences in parental investment in response to parent-absent calls. Ethology 120:258-265.

Lee K.A., M. Wikelski, W.D. Robinson, T.R. Robinson, and K.C. Klasing. 2008. Constitutive immune defences correlate with life-history variables in tropical birds. J Anim Ecol 77: 356-363.

Lobato E., S. Merino, J. Morales, G. Tomás, J. Martínez-de la Puente, E. Sánchez, S. García-Fraile, and J. Moreno. 2008. Sex differences in circulating antibodies in nestling pied flycatchers Ficedula hypoleuca. Ibis 150:799-806.

López-Rull I., P. Celis, C. Salaberria, M. Puerta, and D. Gil. 2011. Post-fledging recruitment in relation to nestling plasma testosterone and immunocompetence in the spotless starling. Funct Ecol 25:500-508.

Martin L.B., II, P. Han, J. Lewittes, J.R. Kuhlman, K.C. Klasing, and M. Wikelski. 2006a. Phytohemagglutinin-induced skin swelling in birds: histological support for a classic immunoecological technique. Funct Ecol 20:290-299.

Martin L.B., II, Z.M. Weil, J.R. Kuhlman, and R.J. Nelson. 2006b. Trade-offs within the immune systems of female white-footed mice, Peromyscus leucopus. Funct Ecol 20: 630-636.

Matson K.D., R.E. Ricklefs, and K.C. Klasing. 2005. A hemolysishemagglutination assay for characterizing constitutive innate humoral immunity in wild and domestic birds. Dev Comp Immunol 29:275-286.

Matson K.D., B.I. Tieleman, and K.C. Klasing. 2006. Capture stress and the bactericidal competence of blood and plasma in five species of tropical birds. Physiol Biochem Zool 79: 556-564.

Maxwell M.H. and G.W. Robertson. 1998. The avian heterophil leucocyte: a review. World's Poult Sci J 54:155-178.

Merino S., J. Martínez, A.P. Møller, A. Barbosa, F. de Lope, and F. Rodríguez-Caabeiro. 2001. Physiological and haematological consequences of a novel parasite on the red-rumped swallow Hirundo daurica. Int J Parasitol 31:1187-1193.

Merino S. and J. Potti. 1995. High prevalence of hematozoa in nestlings of a passerine species, the pied flycatcher (Ficedula hypoleuca). Auk 112:1041-1043.

Møller A.P., G. Sorci, and J. Erritzøe. 1998. Sexual dimorphism in immune defense. Am Nat 152:605-619.

Moreno J., J. Potti, P. Yorio, and P.G. Borboroglu. 2001. Sex differences in cell-mediated immunity in the Magellanic penguin Spheniscus magellanicus. Ann Zool Fenn 38:111-116.

Moreno J., J.P. Veiga, P.J. Cordero, and E. Minguez. 1999. Effects of paternal care on reproductive success in the polygynous spotless starling Sturnus unicolor. Behav Ecol Sociobiol 47:47-53.

Müller W., K. Deptuch, I. López-Rull, and D. Gil. 2007. Elevated yolk androgen levels benefit offspring development in a between-clutch context. Behav Ecol 18:929-936.

Müller W., C. Dijkstra, and T.G.G. Groothuis. 2003. Intersexual differences in T-cell-mediated immunity of blackheaded gull chicks (Larus ridibundus) depend on the hatching order. Behav Ecol Sociobiol 55:80-86.
Müller W., C.M. Eising, C. Dijkstra, and T.G.G. Groothuis. 2004. Within-clutch patterns of yolk testosterone vary with the onset of incubation in black-headed gulls. Behav Ecol 15:893-897.

Müller W., T.G.G. Groothuis, A. Kasprzik, C. Dijkstra, R.V. Alatalo, and H. Siitari. 2005. Prenatal androgen exposure modulates cellular and humoral immune function of blackheaded gull chicks. Proc R Soc B 272:1971-1977.

Muriel J., L. Pérez-Rodríguez, M. Puerta, and D. Gil. 2013. Differential effects of yolk testosterone and androstenedione in embryo development and nestling growth in the spotless starling (Sturnus unicolor). Gen Comp Endocrinol 194:175182 .

. 2015a. Diverse dose-response effects of yolk androgens on embryo development and nestling growth in a wild passerine. J Exp Biol 218:2241-2249.

Muriel J., P. Salmón, A. Nunez-Buiza, F. de Salas, L. PérezRodríguez, M. Puerta, and D. Gil. 2015b. Context-dependent effects of yolk androgens on nestling growth and immune function in a multibrooded passerine. J Evol Biol 28:14761488.

Navara K.J., G.E. Hill, and M.T. Mendonça. 2005. Variable effects of yolk androgens on growth, survival, and immunity in eastern bluebird nestlings. Physiol Biochem Zool 78:570578.

- 2006. Yolk testosterone stimulates growth and immunity in house finch chicks. Physiol Biochem Zool 79: 550-555.

Owen-Ashley N.T., D. Hasselquist, and J.C. Wingfield. 2004. Androgens and the immunocompetence handicap hypothesis: unraveling direct and indirect pathways of immunosuppression in song sparrows. Am Nat 164:490-505.

Palacios M.G., J.E. Cunnick, D. Vleck, and C.M. Vleck. 2009. Ontogeny of innate and adaptive immune defense components in free-living tree swallows, Tachycineta bicolor. Dev Comp Immunol 33:456-463.

Palacios M.G., J.E. Cunnick, D.W. Winkler, and C.M. Vleck. 2007. Immunosenescence in some but not all immune components in a free-living vertebrate, the tree swallow. Proc R Soc B 274:951-957.

. 2012. Interrelations among immune defense indexes reflect major components of the immune system in a freeliving vertebrate. Physiol Biochem Zool 85:1-10.

Partecke J. and H. Schwabl. 2008. Organizational effects of maternal testosterone on reproductive behavior of adult house sparrows. Dev Neurobiol 68:1538-1548.

Peris S.J. 1980. Biología del estornino negro (Sturnus unicolor Temm.): 1. Alimentación y variación de la dieta. Ardeola 25:207-240.

Pigeon G., M. Belisle, D. Garant, A.A. Cohen, and F. Pelletier. 2013. Ecological immunology in a fluctuating environment: an integrative analysis of tree swallow nestling immune defense. Ecol Evol 3:1091-1103.

Pilz K.M., M. Quiroga, H. Schwabl, and E. Adkins-Regan. 2004. European starling chicks benefit from high yolk testosterone levels during a drought year. Horm Behav 46:179-192. 
Pitala N., S. Ruuskanen, T. Laaksonen, B. Doligez, B. Tschirren, and L. Gustafsson. 2009. The effects of experimentally manipulated yolk androgens on growth and immune function of male and female nestling collared flycatchers Ficedula albicollis. J Avian Biol 40:225-230.

R Development Core Team. 2015. R: a language and environment for statistical computing. R Foundation for Statistical Computing, Vienna, Austria.

Roberts M.L., K.L. Buchanan, and M.R. Evans. 2004. Testing the immunocompetence handicap hypothesis: a review of the evidence. Anim Behav 68:227-239.

Rubolini D., M. Romano, R. Martinelli, B. Leoni, and N. Saino. 2006a. Effects of prenatal yolk androgens on armaments and ornaments of the ring-necked pheasant. Behav Ecol Sociobiol 59:549-560.

Rubolini D., M. Romano, R. Martinelli, and N. Saino. 2006 b. Effects of elevated yolk testosterone levels on survival, growth and immunity of male and female yellow-legged gull chicks. Behav Ecol Sociobiol 59:344-352.

Rutkowska J., M. Cichoń, M. Puerta, and D. Gil. 2005. Negative effects of elevated testosterone on female fecundity in zebra finches. Horm Behav 47:585-591.

Rutkowska J., T. Wilk, and M. Cichoń. 2007. Androgendependent maternal effects on offspring fitness in zebra finches. Behav Ecol Sociobiol 61:1211-1217.

Ruuskanen S., B. Doligez, B. Tschirren, N. Pitala, I. Gustafsson, T.G.G. Groothuis, and T. Laaksonen. 2009. Yolk androgens do not appear to mediate sexual conflict over parental investment in the collared flycatcher Ficedula albicollis. Horm Behav 55: 514-519.

Saino N., R.P. Ferrari, M. Romano, R. Martinelli, A. Lacroix, D. Gil, and A.P. Møller. 2006. Maternal allocation of androgens and antagonistic effects of yolk androgens on sons and daughters. Behav Ecol 17:172-181.

Salaberria C., J. Muriel, M. de Luna, D. Gil, and M. Puerta. 2013. The PHA test as an indicator of phagocytic activity in a passerine bird. PLoS ONE 8(12):e84108. doi:10.1371/journal .pone.0084108.

Salvante K.G. 2006. Techniques for studying integrated immune function in birds. Auk 123:575-586.

Sandell M.I., M. Tobler, and D. Hasselquist. 2009. Yolk androgens and the development of avian immunity: an experiment in jackdaws (Corvus monedula). J Exp Biol 212:815-822.

Schwabl H. 1993. Yolk is a source of maternal testosterone for developing birds. Proc Natl Acad Sci USA 90:11446-11450.

Smits J.E., G.R. Bortolotti, and J.L. Tella. 1999. Simplifying the phytohaemagglutinin skin-testing technique in studies of avian immunocompetence. Funct Ecol 13:567-572.

Sorci G., J.J. Soler, and A.P. Møller. 1997. Reduced immunocompetence of nestlings in replacement clutches of the European magpie (Pica pica). Proc R Soc B 264:1593-1598.
Stadecker M.J., M. Lukic, A. Dvorak, and S. Leskowitz. 1977. The cutaneous basophil response to phytohemagglutinin in chickens. J Immunol 118:1564-1568.

Strasser R. and H. Schwabl. 2004. Yolk testosterone organises behavior and male plumage coloration in house sparrows (Passer domesticus). Behav Ecol Sociobiol 56:491-497.

Svobodova M., K. Weidinger, L. Peske, P. Volf, J. Votypka, and P. Vorisek. 2015. Trypanosomes and haemosporidia in the buzzard (Buteo buteo) and sparrowhawk (Accipiter nisus): factors affecting the prevalence of parasites. Parasitol Res 114:551-560.

Tobler M., D. Hasselquist, H.G. Smith, and M.I. Sandell. 2010. Short- and long-term consequences of prenatal testosterone for immune function: an experimental study in the zebra finch. Behav Ecol Sociobiol 64:717-727.

Tschirren B. 2015. Differential effects of maternal yolk androgens on male and female offspring: a role for sex-specific selection? PLoS ONE 10(7): e0133673. doi:10.1371/journal.pone.0133673.

Tschirren B., H. Richner, and H. Schwabl. 2004. Ectoparasitemodulated deposition of maternal androgens in great tit eggs. Proc R Soc B 271:1371-1375.

Tschirren B., V. Saladin, P.S. Fitze, H. Schwabl, and H. Richner. 2005. Maternal yolk testosterone does not modulate parasite susceptibility or immune function in great tit nestlings. J Anim Ecol 74:675-682.

Veiga J.P. 2002. Estornino negro-Sturnus unicolor. In L.M. Carrascal and A. Salvador, eds. Enciclopedia virtual de los vertebrados Españoles. Museo Nacional de Ciencias Naturales, Madrid. http://www.vertebradosibericos.org/.

Verhulst S. and J.-A. Nilsson. 2008. The timing of birds' breeding seasons: a review of experiments that manipulated timing of breeding. Philos Trans R Soc B 363:399-410.

Villanúa D., L. Pérez-Rodríguez, C. Gortázar, U. Höfle, and J. Viñuela. 2006. Avoiding bias in parasite excretion estimates: the effect of sampling time and type of faeces. Parasitology 133:251-259.

Vinkler M., H. Bainova, and T. Albrecht. 2010. Functional analysis of the skin-swelling response to phytohaemagglutinin. Funct Ecol 24:1081-1086.

von Engelhardt N., C. Carere, C. Dijkstra, and T.G.G. Groothuis. 2006. Sex-specific effects of yolk testosterone on survival, begging and growth of zebra finches. Proc R Soc B 273:65-70.

von Engelhardt N., R. Henriksen, and T.G.G. Groothuis. 2009. Steroids in chicken egg yolk: metabolism and uptake during early embryonic development. Gen Comp Endocrinol 163:175-183.

Watve M.G. and R. Sukumar. 1995. Parasite abundance and diversity in mammals: correlates with host ecology. Proc Natl Acad Sci USA 92:8945-8949.

Zinke A., B. Schnebel, V. Dierschke, and M. Ryll. 2004. Prevalence and intensity of excretion of coccidial oocysts in migrating passerines on Helgoland. J Ornithol 145:74-78. 